Could a Cascade of Overwhelming Disseminated Intravascular Coagulation (DIC) be the Primary Pathology in Life Threatening COVID-19 Infections?

\section{Omar S Mansour*}

Consultant Colorectal and Laparoscopic General Surgeon, Assistant Professor and Clinical Lecturer in General Surgery, Department of Surgery, School of Medicine, Al Balqa Applied University, Al Salt, Jordan

*Corresponding Author: Omar S Mansour, Consultant Colorectal and Laparoscopic General Surgeon, Assistant Professor and Clinical Lecturer in General Surgery, Department of Surgery, School of Medicine, Al Balqa Applied University, Al Salt, Jordan.
Received: May 07, 2020

Published: May 27, 2020

(C) All rights are reserved by $\mathbf{0 m a r} \mathbf{S}$

Mansour.

\begin{abstract}
Background: SARS COVID 19 (SARS-CoV-2) is well known to cause severe respiratory distress. It has been recently discovered to be associated with multiple organ dysfunction syndrome (MODS) and disseminated intravascular coagulation (DIC).

Case Report: A previously healthy 53 year old male patient presented to the emergency department (ER) with fever, generalised body aches and epigastric abdominal pain. The patient arrived independently the emergency department. He was not complaining of any headache or respiratory distress. His vitals showed tachycardia, hypotension and hypoxia on room air. Routine blood tests showed acute kidney injury, thrombocytopenia and coagulopathy (elevated prothrombin time ratio and INR) and elevated D-dimers. The patient was fast tracked for ICU admission despite him being vitally stable. He tested positive for COVID-19 infection and was diagnosed to have developed disseminated intravascular coagulation (DIC). The patient deteriorated quickly with seizures and passed away ten hours post arrival to the hospital.

Conclusion: SARS COVID-19 virus can present with atypical symptoms like fever, body aches and seizures, without any significant respiratory symptoms or any severe pulmonary changes on CT scans. COVID-19 infection can also result in severe DIC. High index of suspicion and immediate COVID-19 testing should be used in any patient presenting with fever, body aches or any atypical symptoms. Intensive treatment of the COVID-19 infection and the underlying DIC are crucial in trying to reduce mortality.

Keywords: COVID-19; SARS-CoV-2; Disseminated Intravascular Coagulation (DIC); Acute Respiratory Distress Syndrome (ARDS); Multiple Organ Dysfunction Syndrome (MODS); Coagulopathy
\end{abstract}

\section{Background}

SARS COVID 19 (SARS-CoV-2) is well known to cause severe respiratory distress. It has been recently discovered to be associated with multiple organ dysfunction syndrome (MODS) and disseminated intravascular coagulation (DIC).

\section{Case Report}

A previously healthy 53 year old male patient presented to the emergency department acutely with fever, generalised body aches and epigastric abdominal pain. The fever and body aches started six days prior to hospital presentation and were not responding to antipyretics or non-steroidal anti-inflammatory drugs (NSAIDs). The body aches were worsening over the previous 24 hours. The epigastric pain started 3 days before arrival at the hospital. The patient arrived independently walking to the emergency department. He was not complaining of any headache, shortness of breath or any respiratory distress.
The patient's background history showed that he was working as a staff at a local hotel which was used by the government for quarantining patients recently arrived from countries with high levels of COVID-19 infection rates. This was in lines with WHO guidelines.

Because the patient had been complaining of fever and body aches for six days prior to this presentation, he had been seen by three paramedical and medical teams at home who diagnosed him with muscle spasm and nonspecific headache. Diclofenac sodium injection probably with steroid injections was given to the patient (at least once) by the paramedics at home. The patient did not have any runny nose, cough, shortness of breath or chest pain at any stage.

On arrival, his vital parameter measurements showed tachycardia, hypotension and hypoxia on room air. They were as follows: heart rate $110 \mathrm{BMM}$, blood pressure $70 / 40 \mathrm{mmHg}$, respiratory rate $20 \mathrm{CPM}$, oxygen saturation $90 \%$ on room air. The patient was resuscitated using the $\mathrm{ABC}$ protocol. 
The patient was conscious and well oriented in time and place for the first 5 hours of his presence in the emergency room. His Glasgow coma scale (GCS) was 15/15 on arrival and for the first 5 hours post admission.

Examination of the throat showed mild congestion, his nose had no congestion.

Examination and auscultation of the chest showed clear chest, normal vesicular breathing sounds with no wheezes or crackles. Abdominal examination showed a soft lax abdomen with mild epigastric tenderness and auscultation showed active bowel sounds. There were no signs of peritonitis like guarding, rigidity or rebound tenderness.

Three hours post arrival, the patient started to become severely distressed with generalised body pain and aches. He was shouting from the severity of the body aches.

He had a complete blood count (CBC) test done that showed HB 15.5, white blood cells (WBC) 3.97, Neutrophils (92\%). Lymphocyte: $0.20 \%$, mono: $1.8 \%$ ). The Platelet count was severely decreased at 40 .

Creatinine was quite elevated at $4.24 \mathrm{mg} / \mathrm{dL}$, urea $125 \mathrm{mg} / \mathrm{dL}$ (elevated), uric acid $10.5 \mathrm{mg} / \mathrm{dL}$, Sodium (Na) $134 \mathrm{mmol} / \mathrm{L}$, Potassium (K) $3.6 \mathrm{~mol} / \mathrm{L}$, Chloride (Cl) $99 \mathrm{mmol} / \mathrm{L}$.

Because of the decreased oxygen saturation on oximetry, an arterial blood gas was sent that showed $\mathrm{PH}: 7.37, \mathrm{PCO}_{2}: 17.9 \mathrm{mmHg}$ (decreased, normal 35 - 45), $\mathrm{PO}_{2}: 56 \mathrm{mmHg}$ (decreased, normal 80 - 100), $\mathrm{HCO}_{3}: 10.2 \mathrm{mEq} / \mathrm{L}$ (decreased, normal 22 - 26).

A urine sample was sent for urine analysis and showed: Nitrites negative, leukoesterase negative, WBC 3 , RBC 14, Granular cast positive, protein +1 .

Because the patient presented with epigastric pain and acute kidney injury (AKI), abdominal and renal ultrasounds were done. These scans did not show any obvious abdominal or renal pathologies to explain the patient's epigastric pain or AKI.

Five hours later, and because the patient's severe body aches, low oxygen saturation, hypotension and AKI, a decision was made to admit the patient to the intensive care unit (ICU) for multi organ support and observation. On initial assessment in ICU, the patient was still vitally stable and in the same clinical condition with the same severe generalised pain intensity. His vitals in ICU showed blood pressure $70 / 40 \mathrm{mmHg}$, oxygen saturation $95 \%$ on face mask (4L). The patient was then assessed by multiple medical specialists for these different complaints.

A portable chest X-ray was done in ICU. It did not show any major abnormalities apart form a thick atelectatic band on the right lower zone. Smaller atelectatic bands with mild atelectatic changes were noted in the lower zones bilaterally. The rest of the lungs and costophrenic angles were clear. The heart size was normal (Figure 1).

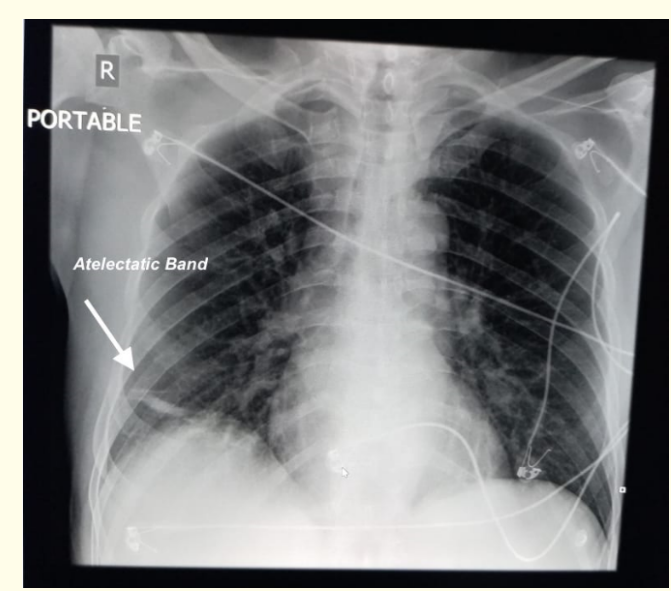

Figure 1: Portable chest X-ray in ICU showing a thick atelectatic band on the right lower zone with mild atelectatic changes bilaterally.

An hour after ICU admission, more blood tests were sent. The results were as follows: C-reactive protein (CRP) 295 (quite elevated), ESR 9, Liver function test: ALT 332 U/L (up to 0.55), AST 442 U/L (up to 0.53), GGT 283 U/L Bilirubin: Total $4.332 \mathrm{umol} / \mathrm{L}$ (up to 20.52), Direct: 3.647 umol/L (up to 5.13), Albumin 28 g/L (52 - 35) Total protein $55 \mathrm{~g} / \mathrm{L}$ (83 - 64), amylase U/L,186 lipase $55 \mathrm{U} / \mathrm{L}, \mathrm{D}-$ dimers $0.45 \mathrm{mcg} / \mathrm{mL}$ (normal < 0.40).

Blood film showed normocytic normochromic RBCs with mild poikilocytosis. WBCs showed Mild leukocytosis and neutrophilia with $41 \%$ band neutrophils. Toxic granulation and some neutrophils showed cytoplasmic vaculation. The platelets were moderately reduced (a large form of platelets was seen). No Schistocytes were seen in general. This blood film suggested bacteria infection (sepsis).

A coagulation profile was done. His INR was 1.67, and the PTT was $37.8 \mathrm{Sec}$. This picture was compatible with DIC probably secondary to sepsis. Since septic shock was suspected, the patient was immediately started on three IV antibiotics, specifically Meropenem, Levofloxacin, and Vancomycin.

Because of his low oxygen saturation and low blood pressures, a cardiac consultation was requested to investigate whether the patient developed pulmonary embolism (PE).

A senior cardiologist reviewed the patient and performed an Echo cardiogram. The echocardiogram showed centric LVH, his left ventricular ejection fraction (EF) was 65 - 70\%, PAP 35. The rest of the echo was normal. Because there were no cardiac or pulmonary causes of his AKI, body aches, hypoxia and hypotension, the cardiologist suggested an urgent testing for Corona Virus. 
After that the patient underwent a high resolution chest computerised tomography (CT) scan. Mild atelectatic changes and bands were seen in the lower lobes. The rest of the lungs were clear. No ground glass opacity or pulmonary nodules. No interstitial thickening or bronchiectatic changes were seen. No pleural effusion or thickening. No hilar, mediastinal masses or adenopathy were seen (Figure 2-5).

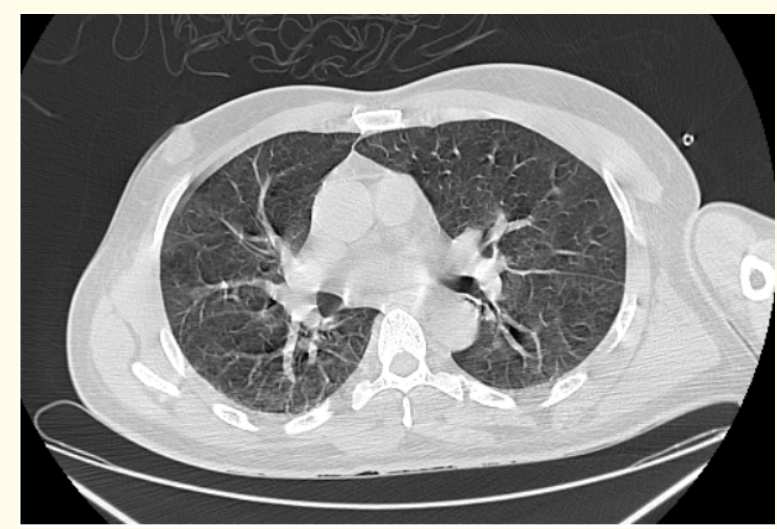

Figure 2: Axial view of thoracic CT scans (lung window). No ground glass opacity or pulmonary nodules. No interstitial thickening or bronchiectatic changes were seen.

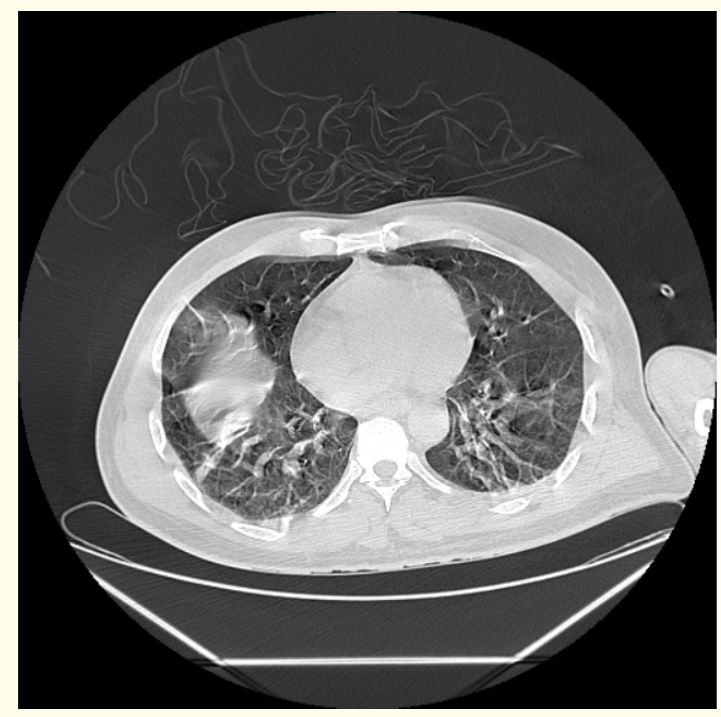

Figure 3: Axial view of thoracic CT scans showing the lower lobes of lungs. Minor consolidation is seen on the right side.

A nasopharyngeal swab for COVID-19 virus PCR was done immediately and sent to the ministry of health COVID-19 labs.

Shortly after admission to ICU, the patient developed a seizure. An urgent Brain computerised tomography (CT) scan was done. No intracerebral focal lesion or extra-axial haematoma was seen. No shift of the midline structures. The ventricular system appeared normal. The grey and white matter differentiation was normal. Normal posterior fossa structures (Figure 6).

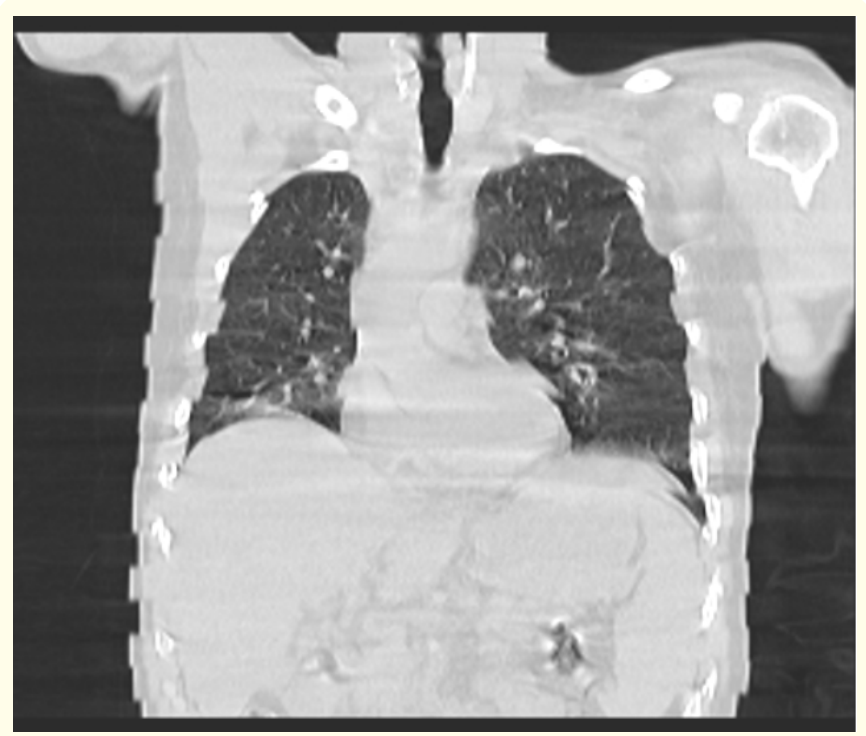

Figure 4: Coronal view of the thoracic CT scan. No major abnormalities were seen on the CT scan.

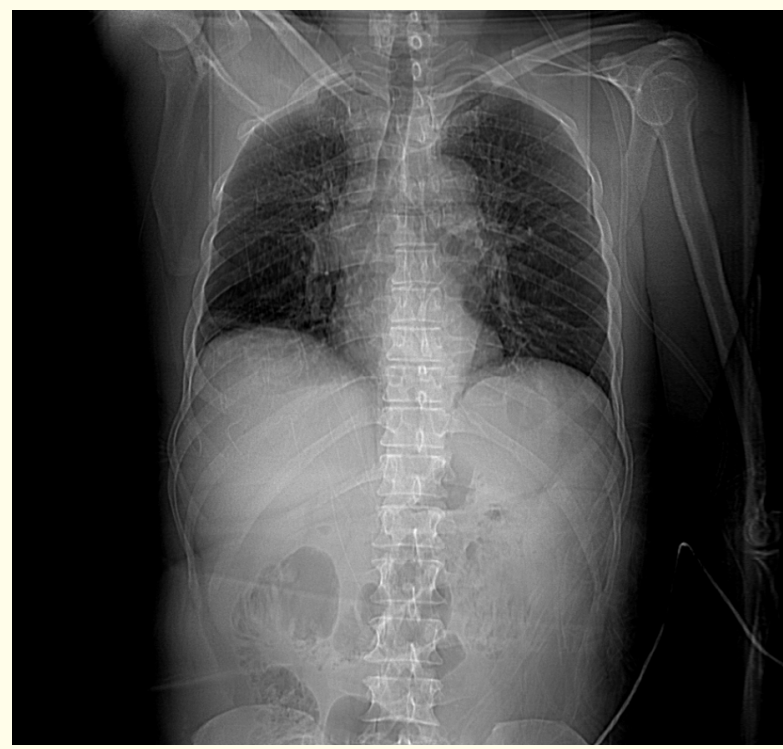

Figure 5: CT Scanogram. No major pathology can be seen on this scan.

Then the patient developed tachypnea and had more recurrent seizures. Because of his deterioration and decreased levels of his level of consciousness, it was decided to intubate him and put him on ventilator. Then he started to loose his vitals and died 10 hours post arrival to the hospital. On the following day, the COVID-19 PCR test came back to be positive.

\section{Discussion}

An outbreak of pneumonia of unknown cause was detected in December 2019, in Wuhan (Hubei, China). It was then quickly determined to be caused by the novel coronavirus, also known as severe acute respiratory syndrome coronavirus 2 (SARS-CoV-2) [1]. 


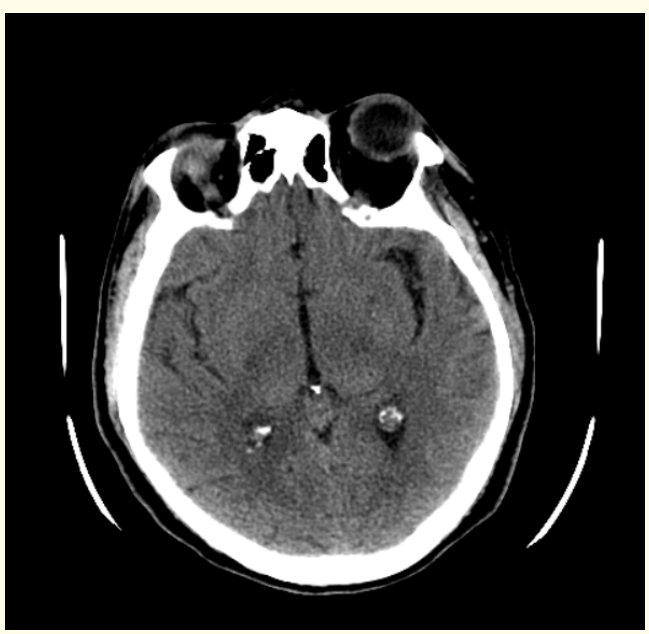

Figure 6a and 6b: Brain CT after the patient developed seizures. No intracerebral focal lesion or extra-axial

haematoma was seen. No shift of the midline structures.

Coronaviruses are a large family of viruses which may cause various types of diseases in humans. They were recognised to also cause illnesses in animals, as well. In humans, several coronaviruses are known to cause respiratory infections ranging from the common cold to more severe diseases such as Severe Acute Respiratory Syndrome (SARS) and Middle East Respiratory Syndrome (MERS). Quite recently, a very contagious type of coronavirus was discovered that causes coronavirus disease COVID-19 [2].

Because of the speed SARS-CoV-2 spreads, the WHO declared this virus to be a pandemic and has called for governments to take "urgent and aggressive action" to change the course of the outbreak.

According to the WHO, the most commonly recognised COVID-19 symptoms are fever, tiredness, and dry cough. Few patients may suffer from aches and pains, nasal congestion, runny nose, sore throat or diarrhoea. These symptoms are usually mild and take place gradually. Some people might become infected but don't develop any symptoms and don't feel unwell. Most people (about $80 \%$ ) recover from the disease without needing special treatment [2]

There is a clinical spectrum of COVID-19 symptoms. They might vary from asymptomatic or pauci-symptomatic forms to clinical conditions characterised by respiratory failure that necessitates mechanical ventilation and support in an intensive care unit (ICU), to multi-organ dysfunctions and systemic manifestations in terms of sepsis, septic shock, and multiple organ dysfunction syndromes (MODS) [3].

The most common symptoms seen in a group of inpatients from Wuhan were fever (40 [98\%] of 41 patients), cough (31 [76\%]), and myalgia or fatigue (18 [44\%]) The less common symptoms recognised were sputum production (11 [28\%] of 39), headache (three [8\%] of 38), haemoptysis (two [5\%] of 39) and diarrhoea (one [3\%] of 38). Dyspnoea developed in 22 (55\%) of 40 patients (median time from illness onset to dyspnoea 8.0 days [IQR 5.0 13.0]). There were 26 (63\%) of 41 patients who developed lymphopenia [4].

In the same Wuhan cohort, most patients presented with fever, dry cough, dyspnoea, and bilateral ground-glass opacities on the chest CT scans. Regarding the consequences of the COVID-19 infection, there were 12 patients (29\%) who had evidence of acute respiratory distress syndrome (ARDS). Only 3 patients (7\%) had acute kidney injury. Chest computerised tomography (CT) of those patients showed pneumonia with abnormal findings in all cases. About a third of those $(13,32 \%)$ required ICU care, and there were $6(15 \%)$ fatal cases [5].

Sepsis is a clinical condition as response to an acute bacterial or viral infection in the blood, with ongoing activation of the immune and coagulation system. Unfortunately, over activation of the coagulation system in acute bacteraemia and viremia can lead to disseminated intravascular coagulation (DIC), microvascular thrombosis-induced hypoxia that contributes to multiorgan failure, septic shock, and death [6,7].

Disseminated intravascular coagulation (DIC) is characterised by systemic activation of blood coagulation, which results in generation and deposition of fibrin, leading to microvascular thrombi in various organs which could result in multiple organ dysfunction syndrome (MODS) [8].

Consumption of clotting factors and platelets in DIC can result in life-threatening haemorrhage [9].

Diagnosis of disseminated intravascular coagulation (DIC) can be difficult, especially in cases of chronic DIC, where clinical and laboratory abnormalities may not be reliable. No single routinely available laboratory test is adequately sensitive or specific to allow a diagnosis of DIC. Multiple commonly available laboratory tests often produce abnormal results in DIC, especially moderateto-severe thrombocytopenia. Besides, the peripheral blood smear can demonstrate evidence of microangiopathic pathology (schistocytes). Scoring systems have been developed to facilitate diagnosis of DIC. Radiological scans are useful only to detect an underlying cause; the diagnosis of DIC is made by combining the clinical impression with any laboratory abnormalities noted [12]

A reduction in the platelet count or a clear downward trend at subsequent measurements is a sensitive (but not specific) sign of DIC. Thrombocytopenia is a feature in up to $98 \%$ of DIC cases with the platelet count $<50 \times 10^{9} / \mathrm{l}$ in approximately $50 \%$ of DIC cases [10].

D-dimer is the degradation product of crosslinked (by factor XIII) fibrin. It reflects ongoing activation of the hemostatic system. The reference concentration of D-dimer is $<250 \mathrm{ng} / \mathrm{mL}$, or $<0.4$ $\mathrm{mcg} / \mathrm{mL}$ [11] 
A low platelet count correlates strongly with markers of thrombin generation, because thrombin-induced platelet aggregation is mainly responsible for platelet consumption [13].

Multiple medical conditions can lead to the development of disseminated intravascular coagulation either through a systemic inflammatory response or the release of procoagulants into the bloodstream. The pathological process of DIC has been estimated to occur in up to $30 \%$ to $50 \%$ of cases of severe sepsis, which is the most common cause of DIC $[14,15]$.

Disseminated intravascular coagulation can lead to multiple complications including:

- Ischaemia: Insufficient blood flow (and subsequent lack of oxygen delivery) to tissue.

- Infarction: Tissue death (by necrosis) due to inadequate blood supply damage to vital organs e.g. brain, lungs, liver, and kidneys [16].

Patients with DIC are at a high risk of venous thromboembolic (VTE) events due to one or more risk factors, including advanced age, recent surgery, immobilisation, in-dwelling vascular catheters and previous VTE history [17].

Venous thromboembolic (VTE) prophylaxis using unfractionated heparin (UFH), low molecular weight heparin (LMWH), and mechanical methods has become standard care in patients with DIC [18].

The association between viral infection and DIC had been thoroughly investigated in the HIV patients. HIV infection is associated with activation of the coagulation cascade and leads to TF expression on CD14+ CD16- CCR2+ monocytes. This is associated with a higher cardiovascular risk in controlled HIV infection when D-dimer is increased [19].

In our case, the patient had a very low platelet count in combination with elevated partial thromboplastin time (PTT) and INR. The INR is derived from prothrombin time (PT) which is calculated as a ratio of the patient's PT to a control PT standardised for the potency of the thromboplastin reagent. This patient was very likely suffering from an overwhelming DIC secondary to sepsis and severe COVID-19 infection. But this patient did not have the commonly recognised COVID-19 related respiratory symptoms but he had mildly decreased oxygen saturation. The exclusion of severe respiratory infection and ARDS was confirmed on the portable chest X-Ray and the thoracic CT scan. These chest scans only showed mild atelectatic bands bi-basally.

Despite the patient having multiple seizures, his brain CT scan did not show any intracranial or cerebral pathology at all that would explain the severe neurological deterioration. The patient also complained of epigastric pain but abdominal ultrasound was normal. Besides, no renal pathology was seen on the ultrasound Finally, his CRP was significantly elevated at 295 confirming a significant inflammatory process in place.

\section{Conclusion}

Two main conclusions can be drawn from this interesting case. The first is that COVID-19 infection may present with atypical symptoms. The second conclusion is an overwhelming DIC can result from severe COVID-19 infection which can be fatal.

High index of suspicion and immediate COVID-19 testing should be used in any patient presenting with fever, body aches or any atypical symptoms.

COVID-19 patients can look well despite having an severe underlying complicated infection.

SARS COVID-19 virus can present with atypical symptoms like fever, aches and seizures, without any noticeable respiratory symptoms or any severe pulmonary changes on CT scan.

Patients presenting with COVID-19 infection should be tested and assessed for an underlying DIC. Vigorous treatment of the infection and any resulting DIC could be the key to successful management of these combined potentially life treating conditions.

In many cases the DIC will spontaneously resolve when the underlying disorder is properly managed. The COVID-19 virus can cause multiple organ dysfunction syndrome (MODS) probably secondary to DIC. This can be a key factor to the successful treatment of any life threatening COVID-19 infection.

Further research is needed to pinpoint the exact pathological mechanisms of action of the COVID-19 virus and its relationship with disseminated intravascular coagulation (DIC).

\section{Conflicts of Interest}

No conflicts of interest.

\section{Bibliography}

1. WHO statement regarding cluster of pneumonia cases in $\mathrm{Wu}$ han, China (2020).

2. Q\&A on coronaviruses (COVID-19).

3. Marco Cascella., et al. "Features, Evaluation and Treatment Coronavirus (COVID-19)". Treasure Island (FL): Stat Pearls (2020).

4. Li X., et al. "Clinical features of patients infected with 2019 novel coronavirus in Wuhan, China”. Lancet 395.10223 (2020): 497-506.

5. Huang C., et al. "Clinical features of patients infected with 2019 novel coronavirus in Wuhan, China”. Lancet 395.10223 (2020): 497-506. 
6. Goeijenbier M., et al. "Review: viral infections and mechanisms of thrombosis and bleeding". Journal of Medical Virology 84 (2012): 1680-1696.

7. Levi M and Van der Poll T. "Coagulation and sepsis". Critical Care Clinics 149 (2017): 38-44.

8. Vincent JL and De Backer D. "Does disseminated intravascular coagulation lead to multiple organ failure?". Critical Care Clinics 21.3 (2005): 469-477.

9. Papageorgiou C., et al. "Disseminated Intravascular Coagulation: An Update on Pathogenesis, Diagnosis, and Therapeutic Strategies". Clinical and Applied Thrombosis/Hemostasis (2018).

10. Spero JA., et al. "Disseminated intravascular coagulation. Findings in 346 patients". Journal of Thrombosis and Haemostasis 43 (1980): 28-33.

11. Pagana KD., et al. "Mosby's Diagnostic and Laboratory Test Reference". 14 ${ }^{\text {th }}$ edition. St. Louis, Mo: Elsevier (2019): 12.

12. Bick RL. "Disseminated intravascular coagulation: objective clinical and laboratory diagnosis, treatment, and assessment of therapeutic response". Seminars in Thrombosis and Hemostasis 22.1 (1996): 69-88.

13. Neame PB., et al. "Thrombocytopenia in septicemia: the role of disseminated intravascular coagulation". Blood 56 (1980): 88-92.

14. Hashimoto D., et al. "Anticoagulant Therapy for Disseminated Intravascular Coagulation After Gastrointestinal Surgery". Anticancer Research 39.1 (2019): 25-31.

15. Hashimoto D., et al. "Anticoagulant Therapy for Disseminated Intravascular Coagulation After Gastrointestinal Surgery". Anticancer Research 39.1 (2019): 25-31.

16. Siegal T., et al. "Clinical and laboratory aspects of disseminated intravascular coagulation (DIC): a study of 118 cases". Thrombosis and Haemostasis 39.1 (1978): 122-134.

17. Cook DJ., et al. "Prevalence, incidence, and risk factors for venous thromboembolism in medical-surgical intensive care unit patients". Journal of Critical Care 20 (2005): 309-313.

18. Samama MM., et al. "A comparison of enoxaparin with placebo for the prevention of venous thromboembolism in acutely ill medical patients. Prophylaxis in medical patients with enoxaparin study group". New England Journal of Medicine 341 (1999): 793-800.

19. Funderburg NT and Lederman MM. "Coagulation and morbidity in treated HIV infection". Thrombosis Research 133.1 (2014): S21-S24.

\section{Assets from publication with us}

- Prompt Acknowledgement after receiving the article

- Thorough Double blinded peer review

- Rapid Publication

- Issue of Publication Certificate

- High visibility of your Published work

Website: www.actascientific.com

Submit Article: www.actascientific.com/submission.php Email us: editor@actascientific.com

Contact us: +919182824667 